\title{
The cd-indices of intervals in the uncrossing partial order on matchings
}

\author{
Younghwan Kim \\ School of Mathematical and Statistical Sciences \\ Arizona State University \\ Tempe, AZ, U.S.A. \\ Younghwan.Kim.2@asu.edu
}

Submitted: Oct 28, 2017; Accepted: Feb 19, 2019; Published: Mar 8, 2019

(C) The author. Released under the CC BY-ND license (International 4.0).

\begin{abstract}
We study flag enumeration in intervals in the uncrossing partial order on matchings. We produce a recursion for the cd-indices of intervals in the uncrossing poset $P_{n}$. We explicitly describe the matchings by constructing an order-reversing bijection. We obtain a recursion for the ab-indices of intervals in the poset $\hat{P}_{n}$, the poset $P_{n}$ with a unique minimum $\hat{0}$ adjoined.
\end{abstract}

Mathematics Subject Classifications: 06A07, 06A11

\section{Introduction}

A graded poset $P$ with a unique maximum and a unique minimum is Eulerian if, for every non-trivial interval $[x, y]$ in $P$, the number of elements of odd rank in $[x, y]$ is equal to the number of elements of even rank in $[x, y]$. One nice property of Eulerian posets is their very simple Möbius functions $\mu_{P}(s, t)=(-1)^{\ell(s, t)}$. Another nice property of Eulerian posets is that they have a cd-index. A cd-index is a non-commutative generating function, which is an efficient way to encode the flag enumeration of Eulerian posets. The cd-index arose from the work of Bayer-Billera [2] on flag $f$-vectors and flag $h$-vectors of Eulerian posets. Bayer-Klapper [3] and Stanley [15] proved the existence of the cd-indices of Eulerian posets. Ehrenborg-Readdy [7] showed the way to obtain the cd-index of some operations, for example, the cd-index of the pyramid over $P$, the product of a poset $P$ with a chain of length one. Reading [13] presented a recursive formula for the cd-indices of intervals in the Bruhat order on a Coxeter group.

The uncrossing partially ordered set $P_{n}$ on matchings is defined on the set of matchings on $2 n$ points on a circle represented with wires, with an order relation: $\tau^{\prime} \leqslant \tau$ in $P_{n}$ if and only if $\tau^{\prime}$ is obtained by resolving a crossing of $\tau$. For example, if $\tau=\{(1,3),(2,4)\}, \tau^{\prime}=$ 
$\{(1,2),(3,4)\}$ and $\tau^{\prime \prime}=\{(1,4),(2,3)\}$ in $P_{2}$ then $\tau^{\prime} \leqslant \tau$ and $\tau^{\prime \prime} \leqslant \tau$. This partial order has been studied by Alman-Lian-Tran [1], Huang-Wen-Xie [8], Kenyon [9], and Lam [12], [11]. The uncrossing posets emerged from studies of circular planar electrical networks. Circular planar networks are finite weighted undirected graphs embedded into a disk, with boundary vertices and interior vertices. By Curtis-Ingerman-Morrow [6] and de VerdièreGitler-Vertigan [5], the electrical networks can be encoded with response matrices. By Lam [12] the space of response matrices for electrical networks has a cell structure, and this cell structure can be described by the uncrossing partial orders (see [12], Proposition 4.10). Let $\hat{P}_{n}$ denote $P_{n}$ with a unique minimum element $\hat{0}$ adjoined. The poset $\hat{P}_{n}$ was conjectured to be Eulerian by Alman-Lian-Tran [1], Huang-Wen-Xie [8], and Kim-Lee [10]. This conjecture was proved by Lam [11]. Thus it is natural for us to pay attention to the cd-index of the posets $\hat{P}_{n}$ and their intervals.

The main result in this work is a recursive formula in Theorem 18 for the cd-indices of intervals in the uncrossing poset $P_{n}$. To find this, we construct an order-reversing bijection in Theorem 10 between $P_{n}$ and $\mathcal{M P}_{n}$, an induced subposet of the group $\tilde{S}_{2 n}$ of affine permutations. We explicitly describe the elements in the induced subposet. Lam [12] introduced a representation of matchings in $P_{n}$ : to a matching $\tau \in P_{n}$, associate $g_{\tau}:[2 n] \rightarrow \mathbb{Z}$ by

$$
g_{\tau}(i)= \begin{cases}\tau(i) & \text { if } i<\tau(i) \\ \tau(i)+2 n & \text { if } i>\tau(i) .\end{cases}
$$

Lam showed that this map $\tau \mapsto g_{\tau}$ identifies $P_{n}$ with an induced subposet of dual Bruhat order of affine permutations of type $(n, 2 n)$ (see [12], Theorem 4.16). However, we need a map to the group $\tilde{S}_{2 n}$ of affine permutations in order to apply a technique in Reading [13] for finding recursions for the cd-indices of intervals in Bruhat order on Coxeter groups. We slightly modify Lam's map $\tau \mapsto g_{\tau}$ to adapt Reading's ideas to our situation. Finally, using the cd-indices of intervals in $P_{n}$, we present a recursion in Theorem 30 for the ab-indices of intervals in the poset $\hat{P}_{n}$.

The paper is structured as follows. In Section 2, we give basic definitions needed to understand this paper: posets, the cd-index, affine permutations, and Bruhat order. In Section 3, the definition of uncrossing posets is presented. In Section 4, we define an induced subposet $\mathcal{M} \mathcal{P}_{n}$ of Bruhat order on affine permutations $\tilde{S}_{2 n}$. We prove that there is an order-reversing bijection between $P_{n}$ and $\mathcal{M P}_{n}$. In Section 5, we recall the recursive formula for the cd-indices of intervals in Bruhat order of a Coxeter group [13]. In Section 6 , we prove that there is a recursive formula for the cd-indices of intervals in $P_{n}$. Moreover, we present a recursive formula for the ab-index of intervals in the poset $\hat{P}_{n}$.

\section{Preliminaries}

In this section we give background information on partially ordered sets, the cd-index, affine permutations, and Bruhat order. The definitions and notations in this section are mainly obtained from Stanley [16] and Björner-Brenti [4]. 


\subsection{Posets}

Let $P$ be a poset and $x, y \in P$. If $x$ covers $y$ we write $x>y$. By an induced subposet, or subposet for short, of $P$, we mean a subset $Q$ of $P$ and a partial ordering on $Q$ such that for $x, y \in Q$ we have $x \leqslant y$ in $Q$ if and only if $x \leqslant y$ in $P$. For $x \leqslant y$ in $P$, a (closed) interval $[x, y]=\{z \in P: x \leqslant z \leqslant y\}$ is a subposet of $P$. Given two posets $P$ and $Q$, form their product $P \times Q$ on the set $\{(x, y): x \in P, y \in Q\}$ such that $\left(x_{1}, y_{1}\right) \leqslant\left(x_{2}, y_{2}\right)$ in $P \times Q$ if $x_{1} \leqslant x_{2}$ in $P$ and $y_{1} \leqslant y_{2}$ in $Q$. A graded poset $P$ with a unique maximum and a unique minimum is Eulerian if, for every interval $[x, y]$ in $P$ where $x<y$, the number of elements of odd rank in $[x, y]$ is equal to the number of elements of even rank in $[x, y]$.

\subsection{The cd-index}

The cd-index arose from the work of Bayer-Billera [2] on flag $f$-vectors of Eulerian posets. They extended the $f$-vector and the $h$-vector of a polytope $\Delta$ to the flag $f$-vector and the flag $h$-vector of the order complex $\Delta(P)$ of a finite graded poset $P$.

Let $\Delta$ be a finite $(d-1)$-dimensional simplicial complex with $f_{i} i$-dimensional faces. The vector $f(\Delta)=\left(f_{0}, f_{1}, \ldots, f_{d-1}\right)$ is called the $f$-vector of $\Delta$. The vector $h(\Delta)=$ $\left(h_{0}, h_{1}, \ldots, h_{d}\right)$, called the $h$-vector, is defined by the relation

$$
\sum_{i=0}^{d} f_{i-1}(x-1)^{d-i}=\sum_{i=0}^{d} h_{i} x^{d-i}
$$

where $f_{-1}=1$ unless $\Delta=\emptyset[2]$.

Let $P$ be a graded poset, rank $n$, with a $\hat{0}$ and a $\hat{1}$. The order complex $\Delta(P)$ of $P$ is defined as follows: the vertices of $\Delta(P)$ are the elements of $P-\{\hat{0}, \hat{1}\}$, and the faces of $\Delta(P)$ are the chains of $P-\{\hat{0}, \hat{1}\}$. The order complex $\Delta(P)$ is a simplicial complex [2]. Stanley [14] enumerated faces of this order complex $\Delta(P)$ as follows. For a chain $C$ in $P-\{\hat{0}, \hat{1}\}$, define $\rho(C)=\{\rho(x): x \in C\}$. Let $[n]:=\{1,2, \ldots, n\}$ and let $2^{[n]}$ be the set of all subsets of $[n]$. For any $S \subseteq[n-1]$, define

$$
\alpha_{P}(S)=\mid\{C \subseteq P: C \text { is a chain such that } \rho(C)=S\} \mid .
$$

We call the function $\alpha_{P}: 2^{[n-1]} \rightarrow \mathbb{N}$ the flag f-vector. We define

$$
\beta_{P}(S)=\sum_{T \subseteq S}(-1)^{|S-T|} \alpha_{P}(T)
$$

The function $\beta_{P}: 2^{[n-1]} \rightarrow \mathbb{N}$ is called the flag h-vector of $P$. Then we can check for the order complex $\Delta=\Delta(P)$

$$
\begin{aligned}
f_{i}(\Delta) & =\sum_{|S|=i+1} \alpha_{P}(S) \\
h_{i}(\Delta) & =\sum_{|S|=i} \beta_{P}(S) .
\end{aligned}
$$


Thus the flag $f$-vector $\alpha_{P}$ of the order complex $\Delta(P)$ counts flags (or chains) of $P-\{\hat{0}, \hat{1}\}$ by length, which are faces of $\Delta(P)$ by dimension, as the $f$-vector counts faces of a polytope by dimension. The flag $h$-vector $\beta_{P}$ plays a role as the $h$-vector of a polytope.

Now we define ab-index of a poset and cd-index of an Eulerian poset. Define the characteristic monomial $u_{S}$ of $S \subseteq[n-1]$ by $u_{S}=e_{1} e_{2} \ldots e_{n-1}$, where

$$
e_{i}= \begin{cases}b & \text { if } i \in S \\ a & \text { if } i \notin S .\end{cases}
$$

Define a noncommutative polynomial $\Psi_{P}(a, b)$, called the ab-index of $P$, by

$$
\Psi_{P}(a, b)=\sum_{S \subseteq[n-1]} \beta_{P}(S) u_{S}
$$

Thus $\Psi_{P}(a, b)$ is a noncommutative generating function for the flag $h$-vector $\beta_{P}$. J. Fine (unpublished) conjectured and Bayer and Klapper [3] proved that if $P$ is an Eulerian poset of rank $n$, then there exists a polynomial $\Phi_{P}(c, d)$ in the noncommutative variables $c$ and $d$ such that $\Psi_{P}(a, b)=\Phi_{P}(a+b, a b+b a)$. The polynomial $\Phi_{P}(c, d)$ is called the $c d$-index of $P$.

\subsection{Affine permutations and Bruhat order}

Let $\tilde{S}_{n}, n \geqslant 2$ be the group of all bijections $w$ of $\mathbb{Z}$ in itself such that

1. $w(x+n)=w(x)+n$ for all $x \in \mathbb{Z}$ and

2. $\sum_{x=1}^{n} w(x)=(1+2+\cdots+n)$,

with composition as group operation. This is the group of affine permutations. The window notation of $w \in \tilde{S}_{n}$ is $w=\left[a_{1}, \ldots, a_{n}\right]$ if $w(i)=a_{i}$ for $i \in[n]$. As a set of generators for $\tilde{S}_{n}$ we take the set of periodic adjacent transpositions $S=\left\{s_{0}, s_{1}, \ldots, s_{n-1}\right\}$ where

$$
\begin{aligned}
s_{1} & :=[2,1,3, \ldots, n] \\
s_{i} & :=[1,2, \ldots, i-1, i+1, i, i+2, \ldots, n] \text { for } 2 \leqslant i \leqslant n-2, \\
s_{n-1} & :=[1,2,3, \ldots, n-2, n, n-1] \\
s_{0} & :=[0,2,3, \ldots, n-1, n+1] .
\end{aligned}
$$

Then, the pair $\left(\tilde{S}_{n}, S\right)$ is a Coxeter system and $\tilde{S}_{n}$ is the Coxeter group of affine permutations of the integers.

There are several partial orders defined on $\tilde{S}_{n}$. We need Bruhat order and from now on, $\tilde{S}_{n}$ denotes the affine permutations, together with this order. For $w \in \tilde{S}_{n}$, a decomposition $w=s_{i_{1}} s_{i_{2}} \ldots s_{i_{k}}$ with letters in $S$ is called a reduced decomposition for $w$ if $k$ is minimal. The word $i_{1} i_{2} \ldots i_{k}$ is called a reduced word for $w$. We say that $k$ is the length of $w$ and denote it by $\ell(w)$. Fix a reduced decomposition for $w=t_{1} t_{2} \ldots t_{k}$ where $t_{i} \in S$ for all $1 \leqslant i \leqslant k$. The Bruhat order is defined by $v \leqslant \leqslant_{B} w$ if and only if there is a reduced subword $t_{i_{1}} t_{i_{2}} \ldots t_{i_{j}}$ of $t_{1} t_{2} \ldots t_{k}$ for $v$ such that $1 \leqslant i_{1}<i_{2}<\cdots<i_{j} \leqslant k$. We will write $v \leqslant w$ for $v \leqslant B$ if there is no possibility of confusion. 


\section{The Uncrossing Posets}

A (complete) matching on $[2 n]$ is a partition of $[2 n]$ of type $(2,2, \ldots, 2)$. Let $P_{n}$ be the set of matchings on $[2 n]$. For a matching $\left\{\left(i_{1}, j_{1}\right), \ldots,\left(i_{n}, j_{n}\right)\right\}$ where $i_{r}<j_{r}$ for all $r$, two blocks $\left(i_{r}, j_{r}\right)$ and $\left(i_{s}, j_{s}\right)$ form a crossing if $i_{r}<i_{s}<j_{r}<j_{s}$. Now resolve any crossing in $\tau$ in either of two ways:
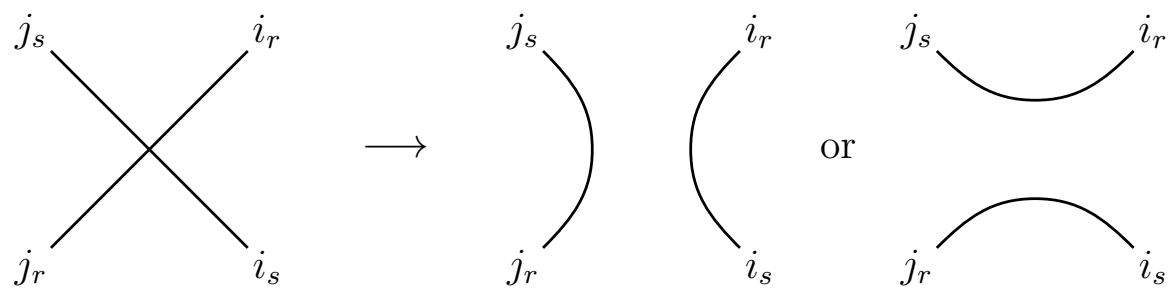

This gives a new matching $\tau^{\prime}$ on $[2 n]$. Then we define the partial order $\tau^{\prime} \leqslant \tau$ on $P_{n}$ if the matching $\tau^{\prime}$ is obtained by resolving a crossing of the matching $\tau$. Let $c(\tau)$ be the number of crossings of $\tau$. The poset $P_{n}$ is graded of rank $\left(\begin{array}{l}n \\ 2\end{array}\right)$ with rank function given by $c(\tau)$. The poset $P_{n}$ has a unique maximum element, namely $\{(1, n+1),(2, n+2), \ldots,(n, 2 n)\}$, and Catalan number $\frac{1}{n+1}\left(\begin{array}{c}2 n \\ n\end{array}\right)$ of minimal elements. Figure 1 is the Hasse diagram of $P_{3}$.

This partial order has been studied by Alman-Lian-Tran [1], Huang-Wen-Xie [8], Kenyon [9], and Lam [12], [11]. The uncrossing posets emerged from studies of circular planar electrical networks, finite weighted undirected graphs embedded into a disk, with boundary vertices and interior vertices. By Curtis-Ingerman-Morrow [6] and de VerdièreGitler-Vertigan [5], the electrical networks can be encoded with response matrices. By Lam [12] the space of response matrices for electrical networks has a cell structure, and this cell structure can be described by the uncrossing partial orders (see [12], Proposition 4.10).

Let $\hat{P}_{n}$ denote $P_{n}$ with a unique minimum element $\hat{0}$ adjoined, where we let $c(\hat{0})=-1$. The poset $\hat{P}_{n}$ was conjectured to be Eulerian by Alman-Lian-Tran [1], Huang-Wen-Xie [8], and Kim-Lee [10], and was proved to be so by Lam [11].

Theorem 1 ([11], Theorem 1). $\hat{P}_{n}$ is an Eulerian poset.

Lam [11] used the map $\tau \mapsto g_{\tau}$ ([12], see (1) in Section 1) as the main tool for proving Theorem 1. Using this map, Lam showed that the number of odd elements equals the number of even rank elements in intervals in $\hat{P}_{n}$. To be specific, he first showed that the number of odd rank elements equals the number of even rank elements in any interval $[\tau, \eta] \subset P_{n}$ by descending induction on $c(\tau)+c(\eta)$. Then he showed the number of odd rank elements equals the number of even rank elements in any interval $[\hat{0}, \eta] \subset \hat{P}_{n}$ by establishing an involution $\sigma \mapsto s_{i} \cdot \sigma$ on the set $\left\{\sigma \in(\hat{0}, \eta] \mid s_{i} \cdot \sigma \neq \sigma\right\}$.

\section{Modular Palindromic Permutations}

Lam [12] mapped matchings $\tau \in P_{n}$ to affine permutations $g_{\tau}$ of type $(n, 2 n)$. See (1) in Section 1. Lam showed that this map $\tau \mapsto g_{\tau}$ identifies $P_{n}$ with an induced subposet of 


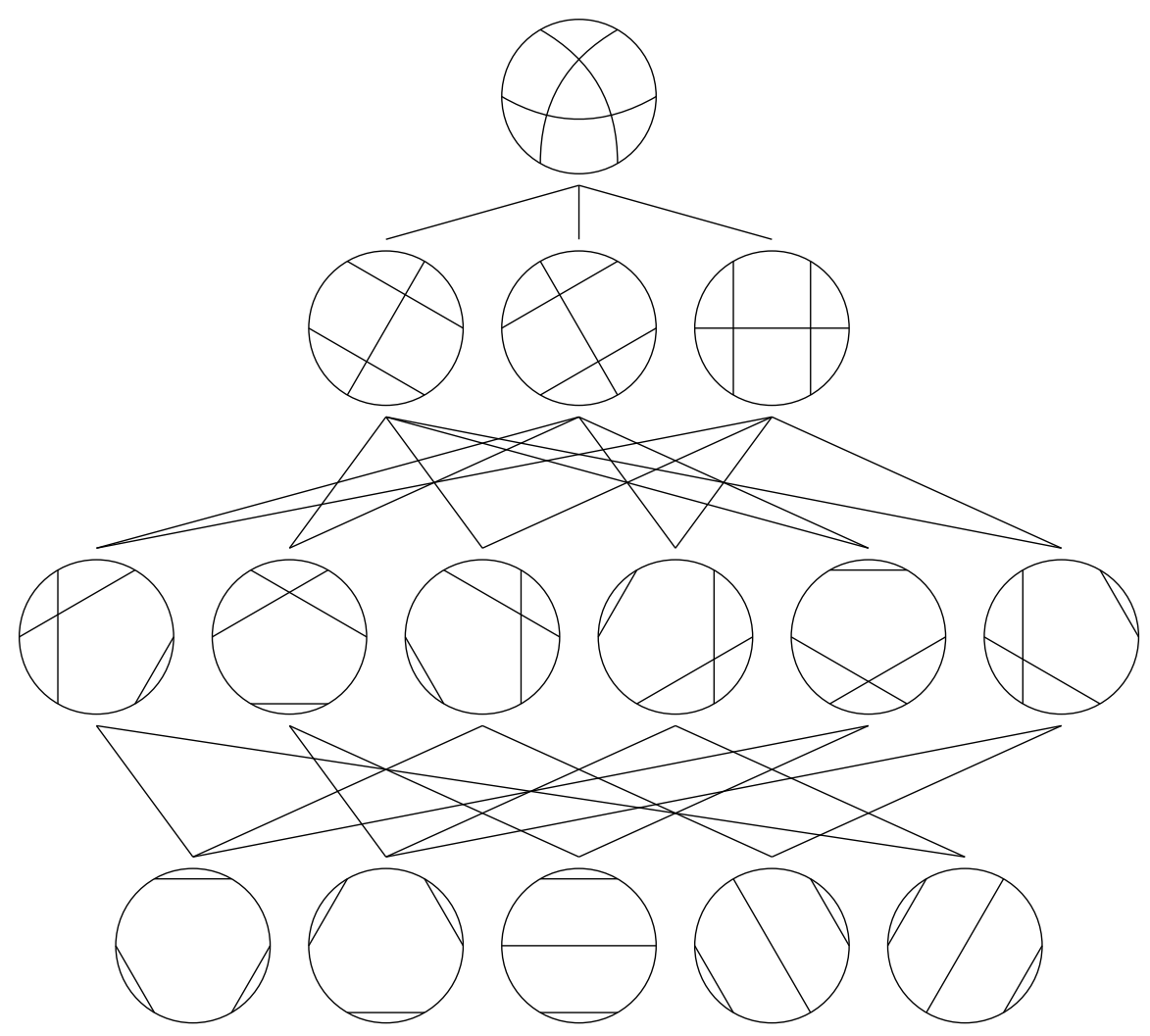

Figure 1: The Hasse diagram of $P_{3}$ (courtesy of Thomas Lam, used with permission)

dual Bruhat order of affine permutations of type $(n, 2 n)$ (Theorem 4.16 in [12]), through Theorem 8.3.7 in Björner-Brenti [4] which characterizes affine Bruhat order in terms of a matrix which tracks inversions.

We notice that if we slightly modify Lam's map it is possible to show that $P_{n}$ is identified with an induced subposet of dual Bruhat order of the group $\tilde{S}_{2 n}$ of affine permutations without Theorem 8.3.7 in [4]. We believe that this identification is necessary to apply a technique in Reading [13] for finding recursions for the cd-indices of intervals in Bruhat order on Coxeter groups. Moreover, we are able to explicitly describe the elements in the induced subposet of affine permutations as modular palindromic permutations (see Theorem 10).

For these reasons, we slightly modify the map $\tau \mapsto g_{\tau}$ to define an injective map $\phi: P_{n} \rightarrow \tilde{S}_{2 n}$ as follows. For a matching $\tau \in P_{n}$, define $h_{\tau}:[2 n] \rightarrow \mathbb{Z}$ by

$$
h_{\tau}(i)= \begin{cases}\tau(i)-n & \text { if } i<\tau(i) \\ \tau(i)+n & \text { if } i>\tau(i) .\end{cases}
$$

Now define $\phi: P_{n} \rightarrow \tilde{S}_{2 n}$ by $\phi(\tau)=\left[h_{\tau}(1), h_{\tau}(2), \ldots, h_{\tau}(2 n)\right]$ in the window notation of the affine permutation group $\tilde{S}_{2 n}$. 
Example 2. Let $\tau=\{(1,4),(2,6),(3,5)\}$ and $\tau^{\prime}=\{(1,3),(2,6),(4,5)\}$ in $P_{3}$. The images of $\tau$ and $\tau^{\prime}$ under Lam's map are $g_{\tau}=\left[g_{\tau}(i)\right]_{i=1}^{6}=[4,6,5,7,9,8]$ and $g_{\tau^{\prime}}=$ $\left[g_{\tau^{\prime}}(i)\right]_{i=1}^{6}=[3,6,7,5,10,8]$. On the other hands, the images under the map $\phi$ are $\phi(\tau)=$ $[1,3,2,4,6,5]=s_{2} s_{5} \in \tilde{S}_{6}$ and $\phi\left(\tau^{\prime}\right)=[0,3,4,2,7,5]=s_{2} s_{0} s_{3} s_{5} \in \tilde{S}_{6}$.

With the map $\phi$, we explicitly describe the subposet of $\tilde{S}_{2 n}$ which is isomorphic to the dual of $P_{n}$. First, we define a modular palindromic permutation and a subset $\mathcal{M P} \mathcal{P}_{n}$ of the affine permutation group $\tilde{S}_{2 n}$.

Definition 3. An affine permutation $w \in \tilde{S}_{2 n}$ is modular palindromic if $w$ has a reduced decomposition $w=s_{i_{1}} s_{i_{2}} \ldots s_{i_{2 k}}$ with $\left|i_{2 k+1-r}-i_{r}\right|=n$ for all $r \in[k]$.

Example 4. Let $\tau=\{(1,4),(2,6),(3,5)\}$ and $\tau^{\prime}=\{(1,3),(2,6),(4,5)\}$ in $P_{3}$ as in Example 2. Both $\phi(\tau)=s_{2} s_{5}$ and $\phi\left(\tau^{\prime}\right)=s_{2} s_{0} s_{3} s_{5}$ are modular palindromic. We also observe that $\phi$ reverses the order, in other words, $\tau^{\prime} \leqslant \tau$ and $\phi\left(\tau^{\prime}\right) \geqslant \phi(\tau)$.

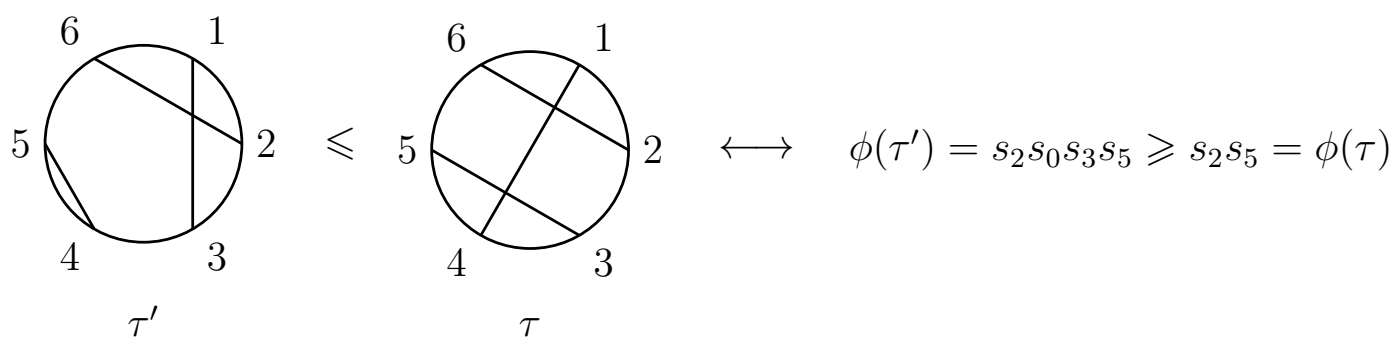

Example 5. Let $n=3$. Let $w=s_{0} s_{5} s_{1} s_{4} s_{2} s_{3} \in \tilde{S}_{6}$. Notice that $w$ is a modular palindromic permutation. The window notation of the permutation is $w=[2,3,7,0,4,5]$. Assume $w=\phi(\tau)$ for some $\tau \in P_{3}$. Since $w(3)=7$, we must have $h_{\tau}(3)=7$, which impossible because $1 \leqslant h_{\tau}(3) \leqslant 5$. Thus $w \notin \phi\left(P_{3}\right)$.

The previous example shows that not all modular palindromic permutations are images of matchings under the map $\phi$. Which conditions are needed for modular palindromic permutations to be images of matchings under the map $\phi$ ? We need the following definition.

Definition 6. The subset $\mathcal{M P} \mathcal{P}_{n}$ of $\tilde{S}_{2 n}$ is the set of all modular palindromic permutations $w \in \tilde{S}_{2 n}$ such that no reduced word of $w$ contains a subword of $n$ consecutive integers.

Notice that $i-n<p(i)<i+n$ for all $i \in[2 n]$ since no reduced decomposition of $p$ contains a subword of $n$ consecutive integers.

Remark 7 . The consecutive integers need not be in adjacent positions in the reduced word.

Now we use $\mathcal{M P}_{n}$ as a poset, equipped with Bruhat order.

Example 8. Let $n=2$. We see that $\mathcal{M P}_{2}$ contains $e, s_{0} s_{2}$ and $s_{1} s_{3}$. Notice that all length four modular palindromic permutations, whose reduced words are 1023, 0132, 2130 and 3201 respectively, have a subword of 2-consecutive integers, and thus $\mathcal{M P}_{2}=$ $\left\{e, s_{0} s_{2}, s_{1} s_{3}\right\}$ and the followings are the Hasse diagrams of $P_{2}$ and the dual of $\mathcal{M P}_{2}$, respectively. 


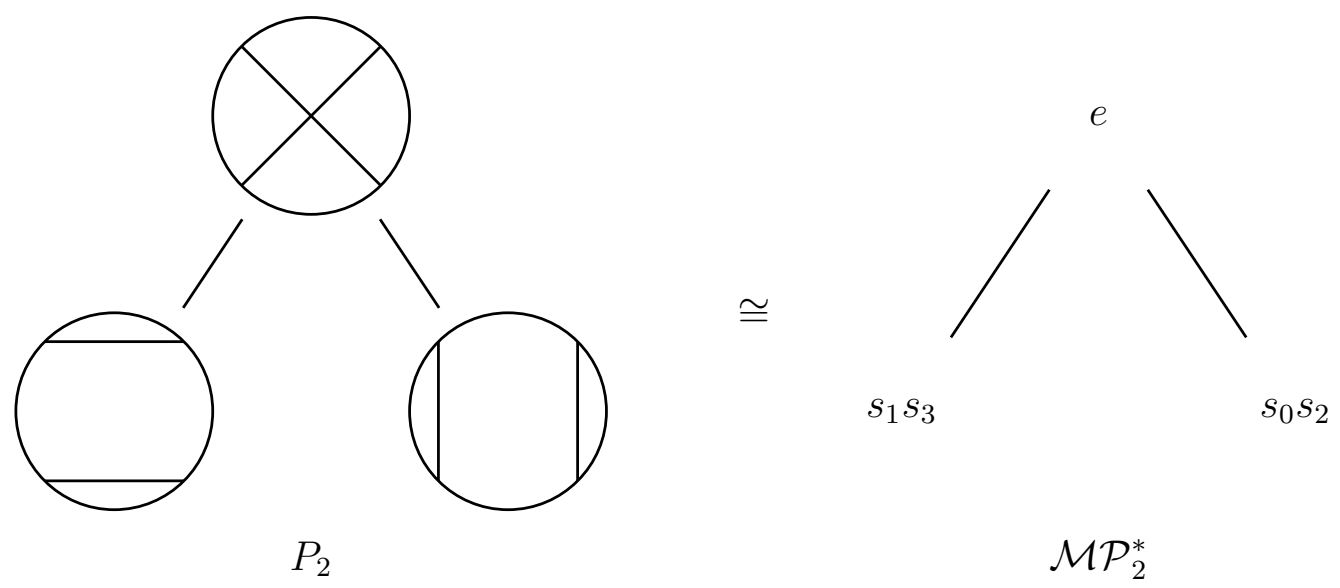

Example 9 (revisited). Let $n=3$. Let $w=s_{0} s_{5} s_{1} s_{4} s_{2} s_{3} \in \tilde{S}_{6}$ with a reduced word 051423. Notice that $w$ is a modular palindromic permutation, which has a subword 543, a 3 consecutive integers. Thus $w \notin \mathcal{M} \mathcal{P}_{3}$.

The following theorem is one of the main results in this paper.

Theorem 10. The map $\phi$ is an order-reversing bijection between $P_{n}$ and $\mathcal{M P}_{n}$.

To prove this theorem, we first state and prove a lemma.

Lemma 11. The image of $P_{n}$ under the map $\phi$ is contained in $\mathcal{M P}_{n}$. In other words, $\phi\left(P_{n}\right) \subseteq \mathcal{M P}_{n}$.

Proof. First, we prove that $\phi(\tau)$ is modular palindromic using decreasing induction on the ranks of $\tau \in P_{n}$.

(i) Base case: the unique maximum element $\hat{1}=\{(1, n+1),(2, n+2), \ldots,(n, 2 n)\}$. Notice that $\phi(\hat{1})=e \in \mathcal{M P}_{n}$ as required.

(ii) Induction step: suppose $\tau \leqslant \hat{1}$ and $\phi(\tau) \in \mathcal{M} \mathcal{P}_{n}$. Choose a crossing generated by a pair of wires $(a, \tau(a))$ and $(b, \tau(b))$ such that $a<b<\tau(a)<\tau(b)$. We can resolve the crossing in two ways.

Case 1: from $\{(a, \tau(a)),(b, \tau(b))\}$ to $\{(a, \tau(b)),(b, \tau(a))\}$. Let $\tau^{\prime} \in \mathcal{M} \mathcal{P}_{n}$ be the matching obtained by resolving the crossing this way.

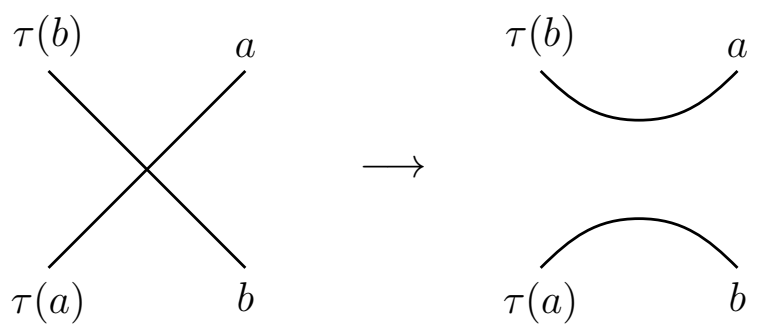

In window notation, we see that

$$
\begin{aligned}
\ldots, \quad a \quad, \ldots, \quad b \quad, \ldots, \tau(a), \ldots, \tau(b), \ldots \\
\phi(\tau)=[\ldots, \tau(a)-n, \ldots, \tau(b)-n, \ldots, a+n, \ldots, b+n, \ldots] \\
\phi\left(\tau^{\prime}\right)=[\ldots, \tau(b)-n, \ldots, \tau(a)-n, \ldots, b+n, \ldots, a+n, \ldots] .
\end{aligned}
$$


Observe that $\phi\left(\tau^{\prime}\right)$ is obtained from $\phi(\tau)$ by swapping the numbers in the $a$-th spot and $b$-th spot and swapping the numbers $a+n$ and $b+n$. Thus, $\phi\left(\tau^{\prime}\right)=t_{a+n, b+n} \phi(\tau) t_{a, b}$ where $t_{a, b}=s_{a} s_{a+1} \ldots s_{b-2} s_{b-1} s_{b-2} \ldots s_{a+1} s_{a}$ is the periodic transposition of $a$ and $b$. Therefore, $\phi\left(\tau^{\prime}\right)$ is modular palindromic.

Case 2: from $\{(a, \tau(a)),(b, \tau(b))\}$ to $\{(a, b),(\tau(a), \tau(b))\}$.
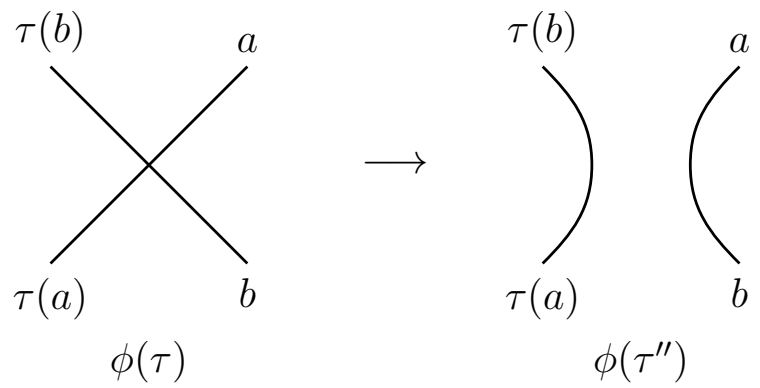

This case can be proved in exactly the same way as the previous case using transpositions $t_{b-n, \tau(a)-n}$ and $t_{b, \tau(a)}$.

Secondly, we prove that there is no reduced word for $\phi(\tau)$ which contains a subword of $n$ consecutive integers. For the sake of contradiction, suppose there is a reduced word for $\phi(\tau)$ containing a subword of $n$ consecutive integers. Without loss of generality, assume the consecutive integers are increasing. Take a maximal length subword $s_{a+1} s_{a+2} \ldots s_{a+m}$ of $\phi(\tau)$ where $m \geqslant n$ and the indices are taken modulo $2 n$ if necessary. Then, observe that $\phi(\tau)(a+m+1)=a+1$, which contradicts that $i-n<\phi(\tau)(i)<i+n$ by the definition of $\phi$ for all $i$.

Corollary 12. The map $\phi$ is order-reversing. In other words, $\tau^{\prime} \leqslant \tau$ in $P_{n}$ implies $\phi\left(\tau^{\prime}\right) \geqslant \phi(\tau)$ in $\mathcal{M P} \mathcal{P}_{n}$ or in $\tilde{S}_{2 n}$.

Proof. By the proof of the previous lemma, we see that if a matching $\tau^{\prime}$ is obtained by resolving a crossing of a matching $\tau$, then $\phi\left(\tau^{\prime}\right)=t_{a, b} \phi(\tau) t_{a+n, b+n}$ for some $a, b \in[2 n]$. Since $\phi(\tau)$ is a subword of $\phi\left(\tau^{\prime}\right)$, we conclude that $\phi(\tau) \leqslant \phi\left(\tau^{\prime}\right)$ as required.

The following lemma will be used when we prove Theorem 10; in particular, we will use the lemma to show that the map $\phi$ is surjective.

Lemma 13. Let $p, q \in \mathcal{M P} \mathcal{P}_{n}$ such that $q=s_{a} p s_{a+n}$ and $\ell(q)=\ell(p)+2$. Let $x, y \in[2 n]$ such that $p(x) \equiv a(\bmod 2 n)$ and $p(y) \equiv a+1(\bmod 2 n)$. Then,

(1) The numbers $x, y, a+n$ and $a+n+1$ are distinct.

(2) $q(i)=p(i)$ for all $i \in[2 n] \backslash\{a+n, a+n+1, x, y\}$.

(3) $q(a+n)=p(a+n+1), q(a+n+1)=p(a+n), q(x) \equiv a+1(\bmod 2 n)$, and $q(y) \equiv a(\bmod 2 n)$. 
Proof. (1) It is clear that $x \neq y$ and $a+n \neq a+n+1$. Since $i-n<p(i)<i+n$ for all $i \in[2 n]$, we know $a<p(a+n)<a+2 n$ and $a+1<p(a+n+1)<a+2 n+1$, and $a+n \neq x$ and $a+n+1 \neq y$. Asume $y=a+n$, then $p(a+n) \equiv a+1(\bmod 2 n)$. Then $p$ should contain a subword of the form $s_{a+1} s_{a+2} \ldots s_{a+n-2} s_{a+n-1}$. Then $q=s_{a} p s_{a+n}$ should contain a subword of $n$ consecutive integers, which contradicts $q \in \mathcal{M P}_{n}$. Assume $x=a+n+1$, then $p(a+n+1) \equiv a(\bmod 2 n)$. Then $p$ should contain a subword of the form $s_{a+2 n+1} s_{a+2 n} \ldots s_{a+n+2} s_{a+n+1}$. Then $q=s_{a} p s_{a+n}$ should contain a subword of $n$ consecutive integers, which contradicts $q \in \mathcal{M} \mathcal{P}_{n}$.

(2) Let $i \in[2 n] \backslash\{a+n, a+n+1, x, y\}$. Then it is clear that $s_{a+n}(i)=i$. Note that $p(i) \notin\{a, a+1\}$ since $i \notin\{x, y\}$, and thus $s_{a}(p(i))=p(i)$. Then we have

$$
q(i)=\left(s_{a} p s_{a+n}\right)(i)=s_{a}(p(i))=p(i) .
$$

(3) Since $s_{a+n}$ acts on the right of $p$ by swapping numbers in the $(a+n)$-th spot and $(a+n+1)$-th spot and $s_{a}$ acts on the left of $p$ by swapping numbers $a$ and $a+1$, we have the third statement.

Proof of Theorem 10. By Lemma 11 and Corollay 12, we need to show that the map $\phi$ is surjective to complete the proof. Let $p \in \mathcal{M P}_{n}$. Since $i-n<p(i)<i+n$ for all $i \in[2 n]$, we define $\tau_{p}$ by

$$
\tau_{p}(i)=\left\{\begin{array}{lll}
p(i)+n & \text { if } & p(i) \leqslant n \\
p(i)-n & \text { if } & p(i) \geqslant n+1
\end{array}\right.
$$

for $i \in[2 n]$. We claim that $\tau_{p}$ is a matching on $[2 n]$. To show $\tau_{p}$ is a matching, we need to show that $\tau_{p}$ is an involution with no fixed point. In other words, we must show that $\tau_{p}(i) \neq i$ and $\tau_{p}^{2}(i)=i$ for all $i \in[2 n]$. Since $i-n<p(i)<i+n$, either $i<\tau_{p}(i)<i+2 n$ or $i-2 n<\tau_{p}(i)<i$, and thus $\tau_{p}(i) \neq i$. Observe that

$$
\tau_{p}^{2}(i)=\left\{\begin{array}{lll}
p(p(i)+n)+n & \text { if } & p(i) \leqslant n \text { and } p(p(i)+n) \leqslant n \\
p(p(i)+n)-n & \text { if } & p(i) \leqslant n \text { and } p(p(i)+n) \geqslant n+1 \\
p(p(i)-n)+n & \text { if } & p(i) \geqslant n+1 \text { and } p(p(i)-n) \leqslant n \\
p(p(i)-n)-n & \text { if } & p(i) \geqslant n+1 \text { and } p(p(i)-n) \geqslant n+1
\end{array}\right.
$$

Since $p$ is an affine permutation in $\tilde{S}_{2 n}$, we see that

$$
p(p(i)-n)+n=p(p(i)+n-2 n)+n=p(p(i)+n)-n
$$

and

$$
p(p(i)-n)-n=p(p(i)+n-2 n)-n=p(p(i)+n)-3 n .
$$

By this observation, we have $\tau_{p}^{2}(i) \equiv p(p(i)+n)+n(\bmod 2 n)$. 
Since $1 \leqslant \tau_{p}(i) \leqslant 2 n$, we can simplify $(2)$ as follows.

$$
\tau_{p}^{2}(i) \equiv p(p(i)+n)+n(\bmod 2 n) \text { and } 1 \leqslant \tau_{p}^{2}(i) \leqslant 2 n .
$$

Now we prove that $\tau_{p}$ is an involution by induction on $\ell(p) / 2$.

Base case: $p=e$. Observe that

$$
e(e(i)+n)+n=e(i+n)+n=(i+n)+n=i+2 n,
$$

and $\tau_{e}^{2}(i)=i$ for all $i \in[2 n]$ as desired.

Induction step: Suppose $\tau_{p}^{2}(i)=i$ for all $i \in[2 n]$ for $p \in \mathcal{M P}_{n}$. Then $p(p(i)+n)+n \equiv$ $i(\bmod 2 n)$, or equivalently, $p(p(i)+n) \equiv i+n(\bmod 2 n)$. Let $q=s_{a} p s_{a+n} \in \mathcal{M} \mathcal{P}_{n}$ with $\ell(q)=\ell(p)+2$. Let $x, y \in[2 n]$ such that $p(x) \equiv a(\bmod 2 n)$ and $p(y) \equiv a+1(\bmod 2 n)$. Let $i \in[2 n] \backslash\{a+n, a+n+1, x, y\}$. By Lemma 13, we see $p(i)+n \not \equiv a+n(\bmod 2 n)$ and $p(i)+n \not \equiv a+n+1(\bmod 2 n)$, and thus

$$
\begin{aligned}
q(q(i)+n)+n & \equiv q(p(i)+n)+n \\
& \equiv\left(s_{a} p s_{a+n}\right)(p(i)+n)+n \\
& \equiv s_{a}(p(p(i)+n)+n \\
& \equiv s_{a}(i+n)+n \\
& \equiv i+2 n,
\end{aligned}
$$

where the last equality is due to $i \notin\{a+n, a+n+1\}$, and hence $\tau_{q}^{2}(i)=i$ for $i \in$ $[2 n] \backslash\{a+n, a+n+1, x, y\}$. We calculate $q(q(i)+n)+n$ for $i \in\{a+n, a+n+1, x, y\}$ as follows.

$$
\begin{aligned}
q(q(a+n)+n)+n & \equiv a+3 n, \\
q(q(a+n+1)+n)+n & \equiv a+3 n+1, \\
q(q(x)+n)+n & \equiv x+2 n, \\
q(q(y)+n)+n & \equiv y+2 n,
\end{aligned}
$$

and thus $\tau_{q}^{2}(i)=i$ for $i \in\{a+n, a+n+1, x, y\}$.

Hence by induction, $\tau_{p}$ is an involution and therefore $\tau_{p}$ is a matching on $[2 n]$. By the construction of $\tau_{p}$, observe that

$$
\phi\left(\tau_{p}\right)(i)=h_{\tau_{p}}(i)= \begin{cases}\tau_{p}(i)-n=(p(i)+n)-n & \text { if } \quad i<\tau_{p}(i) \Leftrightarrow p(i) \leqslant n \\ \tau_{p}(i)+n=(p(i)-n)+n & \text { if } \quad i>\tau_{p}(i) \Leftrightarrow p(i) \geqslant n+1\end{cases}
$$

and thus $\phi\left(\tau_{p}\right)(i)=p(i)$ for all $i \in[2 n]$, which shows that the map $\phi$ is surjective.

\section{The cd-index of the posets $P_{n}$}

In the previous section, we saw that the poset $P_{n}$, without $\hat{0}$ adjoined, is isomorphic to a subposet of the dual Bruhat order of affine permutations. Reading [13] provided a 
recursive formula for the cd-indices of intervals in the Bruhat order on a Coxeter group, and it looks promising to examine the recursion to compute the cd-index of $P_{n}$.

The product of a poset $P$ with a chain of length one is called the pyramid of $P$, and denoted by $\operatorname{Pyr}(P)$. We will use the proposition from Ehrenborg-Readdy [7] which produces the cd-index of $\operatorname{Pyr}(P)$ from the cd-index of $P$.

Proposition 14 ([7], Proposition 4.2). Let $P$ be an Eulerian poset. Then

$$
\Phi_{P y r(P)}=\frac{1}{2}\left(\Phi_{p} \cdot c+c \cdot \Phi_{P}+\sum_{x: 0<<<\hat{1}} \Phi_{[\hat{0}, x]} \cdot d \cdot \Phi_{[x, \hat{1}]}\right) .
$$

A zipper in a poset $P$ is a triple of distinct elements $x, y, z \in P$ such that $\{w: w<$ $x\}=\{w: w<y\}$ and $z=x \vee y$ covers $x$ and $y$ but covers no other element. Please see Figure 2 .
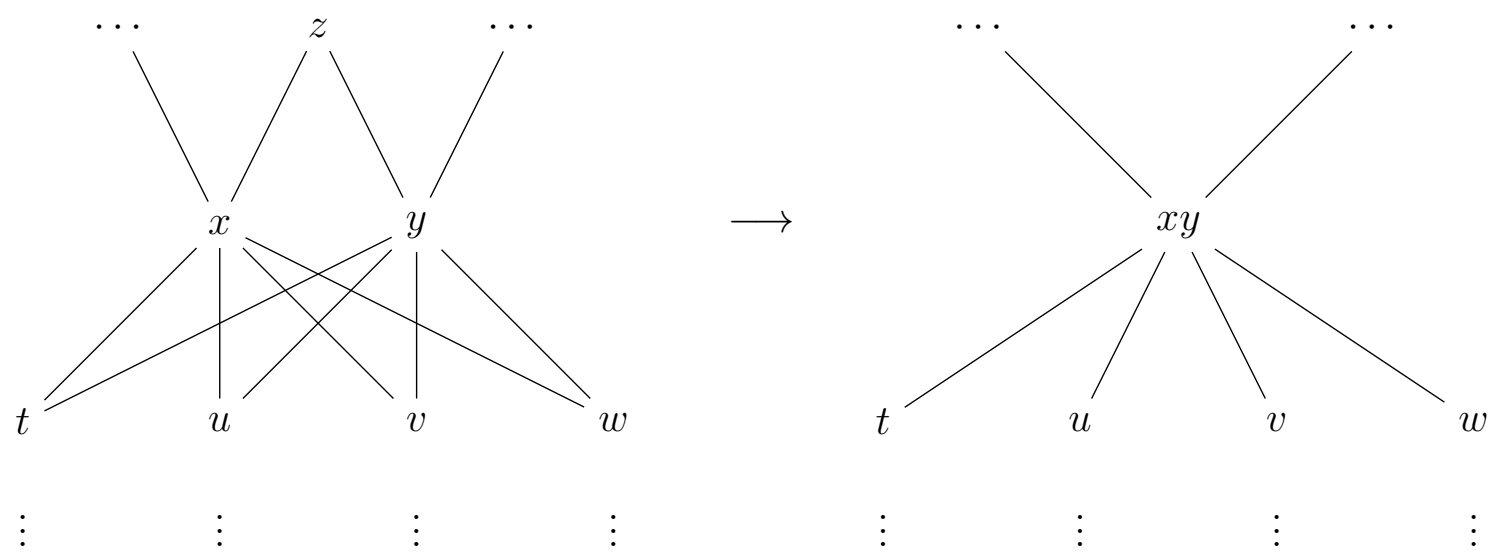

Figure 2: A zipper $\{x, y, z\}$ and zip of the zipper

The operation zip of a zipper $\{x, y, z\} \subset P$ is defined as follows. Let $x y$ be a single new element not in $P$, and define $P^{\prime}=(P-\{x, y, z\}) \cup\{x y\}$, with a binary relation $\preceq$ on $P^{\prime}$, given by:

$$
\begin{aligned}
a \preceq b & \text { if } a \leqslant b \text { in } P-\{x, y, z\} \\
x y \preceq a & \text { if } x \leqslant a \text { or if } y \leqslant a \text { in } P-\{x, y, z\} \\
a \preceq x y & \text { if } a \leqslant x \text { or (equivalently) if } a \leqslant y \text { in } P-\{x, y, z\} \\
x y \preceq x y . &
\end{aligned}
$$

We can think of the operation zip of a zipper $\{x, y, z\}$ as deleting $z$ and identifying $x$ with $y$. The zip operation of a zipper produces a new poset. The zip operation of an Eulerian poset is also Eulerian. Furthermore, the following theorem provides a formula for the cd-index of the resulting poset in terms of the cd-index of the initial Eulerian poset. 
Theorem 15 ([13], Theorem 4.6). $P^{\prime}$ is a poset under the partial order $\preceq$. If $P$ is Eulerian then so is $P^{\prime}$. Moreover, $P$ has a cd-index $\Phi_{P}$ if and only if $P^{\prime}$ has a cd-index $\Phi_{P^{\prime}}$, and

$$
\Phi_{P^{\prime}}=\Phi_{P}-\Phi_{[\hat{0}, x]} \cdot d \cdot \Phi_{[z, \hat{1}]} \cdot
$$

The following theorem is a structural recursion for Bruhat intervals.

Theorem 16 ([13], Theorem 5.5). Let $(W, S)$ be a Coxeter system. Let $w, u \in W$ and $s \in S$. Let $w s>w, u s>u$ and $u \leqslant w$.

(1) If $u s \notin[u, w]$ then $[u, w s] \cong[u, w] \times[1, s]$ and $[u s, w s] \cong[u, w]$.

(2) If us $\in[u, w]$, then $[u, w s]$ can be obtained from $[u, w] \times[1, s]$ by a sequence of zippings.

From these two theorems, Reading [13] produced recursions for the cd-indices of Bruhat intervals. For $v \in W$ and $s \in S$, define $\sigma_{s}(v):=\ell(v s)-\ell(v) \in\{-1,1\}$.

Theorem 17 ([13], Theorem 6.1). Let $(W, S)$ be a Coxeter system. Let $w, u \in W$ and $s \in S$. Let $u<u s, w<w s$ and $u \leqslant w$.

(1) If $u s \notin[u, w]$, then $\Phi_{[u s, w s]}=\Phi_{[u, w]}$, and

$$
\begin{aligned}
\Phi_{[u, w s]} & =\Phi_{\operatorname{Pyr}([u, w])} \\
& =\frac{1}{2}\left(\Phi_{[u, w]} \cdot c+c \cdot \Phi_{[u, w]}+\sum_{v: u<v<w} \Phi_{[u, v]} \cdot d \cdot \Phi_{[v, w]}\right) .
\end{aligned}
$$

(2) If $u s \in[u, w]$, then

$$
\begin{aligned}
\Phi_{[u, w s]} & =\Phi_{P y r([u, w])}-\sum_{\substack{v: u<v<w \\
v s<v}} \Phi_{[u, v]} \cdot d \cdot \Phi_{[v, w]} \\
& =\frac{1}{2}\left(\Phi_{[u, w]} \cdot c+c \cdot \Phi_{[u, w]}+\sum_{v: u<v<w} \sigma_{s}(v) \Phi_{[u, v]} \cdot d \cdot \Phi_{[v, w]}\right) .
\end{aligned}
$$

We claim that there are recurrence relations of the cd-indices of intervals in $\mathcal{M P}_{n}$, and also in $P_{n}$. For $v \in \mathcal{M P} \mathcal{P}_{n}$ and $s=s_{i} \in S$ and $s^{\prime}=s_{i+n} \in S$, define $\sigma_{s}(v):=$ $\frac{1}{2}\left[\ell\left(s^{\prime} s^{\prime}\right)-\ell(v)\right] \in\{-1,1\}$.

Theorem 18. Let $u, w \in \mathcal{M P}_{n}$ and let $s=s_{i}$ and $s^{\prime}=s_{i+n}$ for some $0 \leqslant i<2 n$. Let $u<$ sus $^{\prime}, w<s w s^{\prime}$ and $u \leqslant w$.

(1) If sus' $\notin[u, w]$, then $\Phi_{\left[s u s^{\prime}, s w s^{\prime}\right]}=\Phi_{[u, w]}$, and

$$
\begin{aligned}
\Phi_{\left[u, s w s^{\prime}\right]} & =\Phi_{\operatorname{Pyr}([u, w])} \\
& =\frac{1}{2}\left(\Phi_{[u, w]} \cdot c+c \cdot \Phi_{[u, w]}+\sum_{\substack{v \in \mathcal{M} \mathcal{P}_{n} \\
u<v<w}} \Phi_{[u, v]} \cdot d \cdot \Phi_{[v, w]}\right) .
\end{aligned}
$$


(2) If $s u s^{\prime} \in[u, w]$, then

$$
\begin{aligned}
\Phi_{\left[u, s w s^{\prime}\right]} & =\Phi_{P y r([u, w])}-\sum_{\substack{v \in \mathcal{M} \mathcal{P}_{n} \\
u<v<w \\
s v s^{\prime}<v}} \Phi_{[u, v]} \cdot d \cdot \Phi_{[v, w]} \\
& =\frac{1}{2}\left(\Phi_{[u, w]} \cdot c+c \cdot \Phi_{[u, w]}+\sum_{\substack{v \in \mathcal{M} \mathcal{P}_{n} \\
u<v<w}} \sigma_{s}(v) \Phi_{[u, v]} \cdot d \cdot \Phi_{[v, w]}\right) .
\end{aligned}
$$

Remark 19. We state but do not prove the recurrence relation of the cd-index of the interval $\left[s u s^{\prime}, s w s^{\prime}\right]$ if $s u s^{\prime} \in[u, w]$. In order to prove this, we need a poset operation $S h_{v}$ of vertex shaving (see Chapter 5 in [13] for details).

$$
\begin{aligned}
\Phi_{\left[s u s^{\prime}, s w s^{\prime}\right]} & =S h_{s u s^{\prime}} \Phi_{[u, w]}-\sum_{\substack{v \in \mathcal{M} \mathcal{P}_{n} \\
s u s^{\prime}<v<w \\
s v s^{\prime}<v}} \Phi_{\left[s u s^{\prime}, v\right]} \cdot d \cdot \Phi_{[v, w]} \\
& =\Phi_{[u, w]}+\frac{1}{2}\left(\Phi_{\left[s u s^{\prime}, w\right]} \cdot c-c \cdot \Phi_{\left[s u s^{\prime}, w\right]}+\sum_{\substack{v \in \mathcal{M} \mathcal{P}_{n} \\
s u s^{\prime}<v<w}} \sigma_{s}(v) \Phi_{\left[s u s^{\prime}, v\right]} \cdot d \cdot \Phi_{[v, w]}\right) .
\end{aligned}
$$

Example 20. Let $u=e$ and $w=s_{1} s_{2} s_{5} s_{4}$ in $\mathcal{M P}_{3}$. The Hasse diagrams for intervals $[u, w],\left[s_{3} u s_{0}, s_{3} w s_{0}\right],\left[u, s_{3} w s_{0}\right]$ and $\left[u, s_{2} w s_{5}\right]$ are in Figure 3 .

(1) Let $s=s_{3}$ and $s^{\prime}=s_{0}$. Observe that $u<s u s^{\prime}, w<s w s^{\prime}, u \leqslant w$ and $s u s^{\prime} \notin[u, w]$. We see that $\Phi_{\left[s u s^{\prime}, s w s^{\prime}\right]}=\Phi_{\left[s_{3} s_{0}, s_{3} s_{1} s_{2} s_{5} s_{4} s_{0}\right]}=c=\Phi_{\left[e, s_{1} s_{2} s_{5} s_{4}\right]}=\Phi_{[u, w]}$. Notice that the intervals $[u, w]$ and $\left[s_{3} u s_{0}, s_{3} w s_{0}\right]$ are isomorphic to Boolean lattice $B_{2}$. The cd-index of $B_{2}$ is $\Phi_{B_{2}}(c, d)=c$. We also have

$$
\begin{aligned}
\Phi_{\left[u, s w s^{\prime}\right]} & =\Phi_{\left[e, s_{3} s_{1} s_{2} s_{5} s_{4} s_{0}\right]} \\
& =\frac{1}{2}\left(\Phi_{\left[e, s_{1} s_{2} s_{5} s_{4}\right]} \cdot c+c \cdot \Phi_{\left[e, s_{1} s_{2} s_{5} s_{4}\right]}+\sum_{e<v<s_{1} s_{2} s_{5} s_{4}} \Phi_{[e, v]} \cdot d \cdot \Phi_{\left[v, s_{1} s_{2} s_{5} s_{4}\right]}\right) \\
& =\frac{1}{2}\left(c \cdot c+c \cdot c+\Phi_{\left[e, s_{1} s_{4}\right]} \cdot d \cdot \Phi_{\left[s_{1} s_{4}, s_{1} s_{2} s_{5} s_{4}\right]}+\Phi_{\left[e, s_{2} s_{5}\right]} \cdot d \cdot \Phi_{\left[s_{2} s_{5}, s_{1} s_{2} s_{5} s_{4}\right]}\right) \\
& =\frac{1}{2}\left(c^{2}+c^{2}+d+d\right)=c^{2}+d .
\end{aligned}
$$

Note that the interval $\left[u, s_{3} w s_{0}\right]$ is isomorphic to Boolean lattice $B_{3}$. The cd-index of $B_{3}$ is $\Phi_{B_{3}}(c, d)=c^{2}+d$.

(2) Let $s=s_{2}$ and $s^{\prime}=s_{5}$. Observe that $u<s u s^{\prime}, w<s w s^{\prime}, u \leqslant w$ and $s u s^{\prime} \in[u, w]$. 

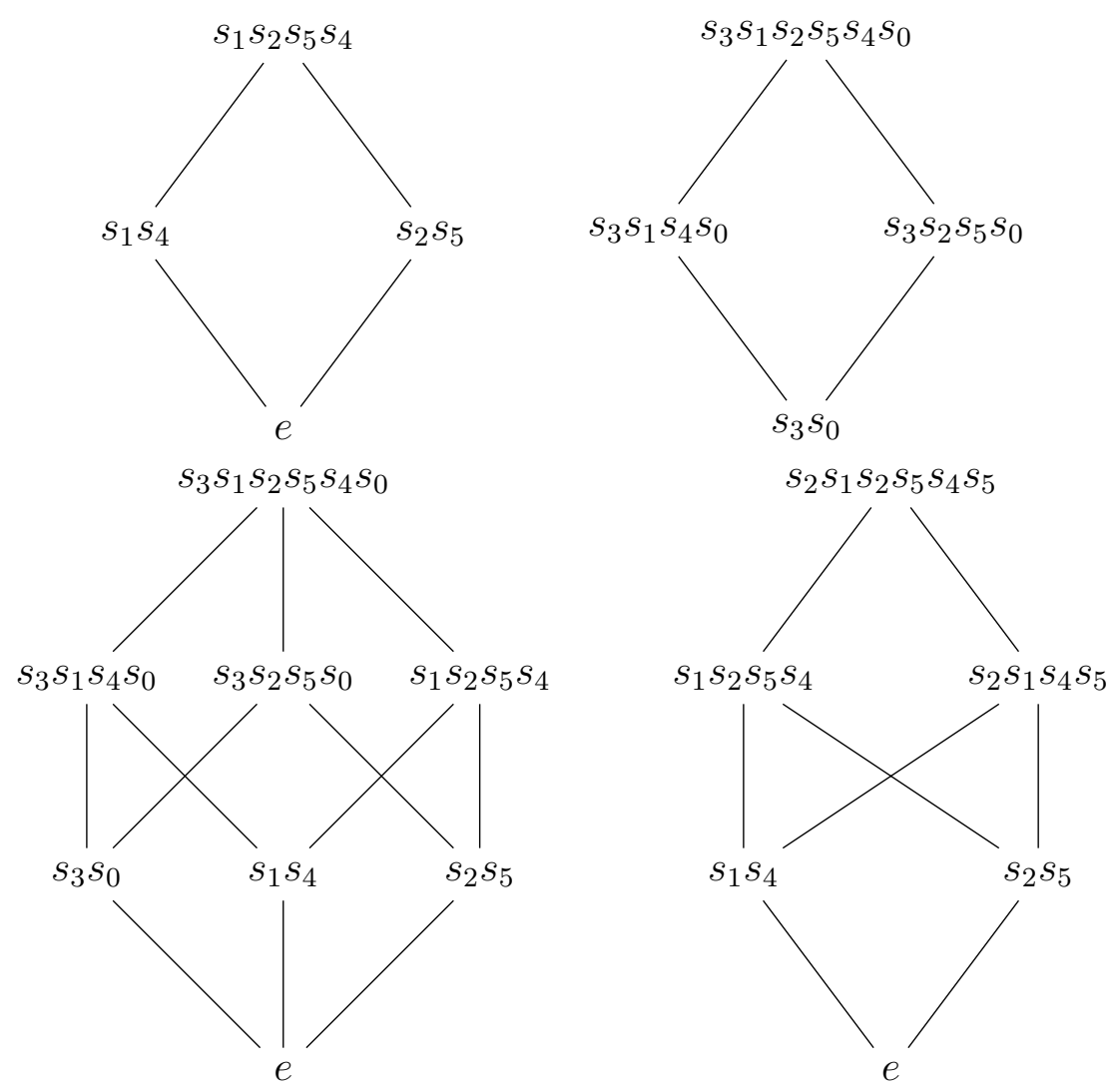

Figure 3: The Hasse diagrams for some intervals in $\mathcal{M P}_{3}$

Note that $\sigma_{s_{2}}\left(s_{1} s_{4}\right)=\frac{1}{2}(4-2)=1$ and $\sigma_{s_{2}}\left(s_{2} s_{5}\right)=\frac{1}{2}(0-2)=-1$. We see that

$$
\begin{aligned}
\Phi_{\left[u, s w s^{\prime}\right]} & =\Phi_{\left[e, s_{2} s_{1} s_{2} s_{5} s_{4} s_{5}\right]} \\
& =\frac{1}{2}\left(\Phi_{\left[e, s_{1} s_{2} s_{5} s_{4}\right]} \cdot c+c \cdot \Phi_{\left[e, s_{1} s_{2} s_{5} s_{4}\right]}+\sum_{e<v<w} \sigma_{s_{2}}(v) \Phi_{[e, v]} \cdot d \cdot \Phi_{\left[v, s_{1} s_{2} s_{5} s_{4}\right]}\right) \\
& =\frac{1}{2}\left(c \cdot c+c \cdot c+\Phi_{\left[e, s_{1} s_{4}\right]} \cdot d \cdot \Phi_{\left[s_{1} s_{4}, s_{1} s_{2} s_{5} s_{4}\right]}-\Phi_{\left[e, s_{2} s_{5}\right]} \cdot d \cdot \Phi_{\left[s_{2} s_{5}, s_{1} s_{2} s_{5} s_{4}\right]}\right) \\
& =\frac{1}{2}\left(c^{2}+c^{2}+d-d\right)=c^{2} .
\end{aligned}
$$

Observe that the interval $\left[u, s_{2} w s_{5}\right]$ is isomorphic to Bruhat order of symmetric group $S_{3}$. The cd-index of Bruhat order of $S_{3}$ is $\Phi_{S_{3}}(c, d)=c^{2}$.

\section{Proof of Theorem 18}

In this section we let $u, w \in \mathcal{M P}_{n}$ and let $s=s_{i} \in \tilde{S}$ and let $s^{\prime}=s_{i+n} \in \tilde{S}$. Suppose $u<s u s^{\prime}$ and $w<s w s^{\prime}$. We slightly modify Reading's [13] map $\eta$ to define a map 
$\eta:[u, w] \times\left[e, s s^{\prime}\right] \rightarrow\left[u, s w s^{\prime}\right]$, as follows:

$$
\begin{aligned}
\eta(v, e) & =v \\
\eta\left(v, s s^{\prime}\right) & = \begin{cases}s v s^{\prime} & \text { if } s v s^{\prime}>v \\
v & \text { if } s v s^{\prime}<v\end{cases}
\end{aligned}
$$

The following proposition is known as Lifting Property of Bruhat order.

Proposition 21 ([4], Proposition 1.2 (Lifting Property of Bruhat order)). Let $(W, S)$ be a Coxeter system. Let $u, w \in W$ and $s \in S$. If $w>w s$ and $u s>u$, then the following are equivalent:

(i) $w>u$

(ii) $w s>u$

(iii) $w>u s$.

We will need the following proposition, which is the analogue of Proposition 21 for $\mathcal{M P}{ }_{n}$

Proposition 22 (Lifting Property of $\mathcal{M P}_{\boldsymbol{n}}$ ). Let $u, w \in \mathcal{M P}_{n}$ and let $s=s_{i} \in \tilde{S}$. Let $s^{\prime}=s_{i+n} \in \tilde{S}$. If $w>s w s^{\prime}$ and sus $^{\prime}>u$, then the following are equivalent:

(i) $w>u$

(ii) $s w s^{\prime}>u$

(iii) $w>$ sus' $^{\prime}$.

Proof. Since $w>s w s^{\prime}$, by transitivity (ii) implies (i). Since $s u s^{\prime}>u$, by transitivity (iii) implies (i). So assume (i) $w>u$. Choose a reduced decomposition for $s w s^{\prime}=t_{1} t_{2} \ldots t_{2 q}$ where $t_{i} \in \tilde{S}$ for all $i \in[2 q]$ such that the reduced word is modular palindromic. Then $w=$ $s t_{1} t_{2} \ldots t_{2 q} s^{\prime}$ is also reduced and modular palindromic. There is a reduced decomposition for $u$

$$
u=t_{i_{1}} t_{i_{2}} \ldots t_{i_{2 r}}
$$

which is a subword of $w=s t_{1} t_{2} \ldots t_{2 q} s^{\prime}$. Since $s u s^{\prime}>u$, we have $t_{i_{1}} \neq s$ and $t_{i_{2 r}} \neq s^{\prime}$, and thus both (ii) $s w s^{\prime}>u$ and (iii) $w>s u s^{\prime}$ hold as desired.

We claim that the map $\eta$ is well-defined. To show this, let $v \in[u, w]$. Because we have assumed $w<s w s^{\prime}$ we know that $v \in[u, w] \subset\left[u, s w s^{\prime}\right]$, so we may assume that $\eta\left(v, s s^{\prime}\right)=s v s^{\prime}$. In this case, $v<s v s^{\prime}$, thus we see $u \leqslant v<s v s^{\prime}<s w s^{\prime}$ where the last inequality is due to the lifting property, and therefore $\eta\left(v, s s^{\prime}\right) \in\left[u, s w s^{\prime}\right]$. The following proposition and Proposition 5.1 in [13] are the same statement on different posets: Bruhat order of Coxeter groups and the induced subposet $\mathcal{M P}_{n}$. Here, we show the order-preserving part of the statement on the induced subposet $\mathcal{M} \mathcal{P}_{n}$. For the proof of the surjective part, see Proposition 5.1 in [13]. 
Proposition 23. If $u<s u s^{\prime}$ and $w<s w s^{\prime}$, then $\eta:[u, w] \times\left[e, s s^{\prime}\right] \rightarrow\left[u, s w s^{\prime}\right]$ is an surjective order-preserving map.

Proof. Suppose $\left(v_{1}, a_{1}\right) \leqslant\left(v_{2}, a_{2}\right)$ in $[u, w] \times\left[e, s s^{\prime}\right]$. Since $e \leqslant a_{1} \leqslant a_{2} \leqslant s s^{\prime}$, so we break up into three cases.

Case 1: $a_{1}=e$ and $a_{2}=e$. Then $\eta\left(v_{1}, a_{1}\right)=v_{1} \leqslant v_{2}=\eta\left(v_{2}, a_{2}\right)$.

Case 2: $a_{1}=e$ and $a_{2}=s s^{\prime}$. Then either $\eta\left(v_{2}, a_{2}\right)=v_{2}$ with $v_{2}>s v_{2} s^{\prime}$ or $\eta\left(v_{2}, a_{2}\right)=$ $s v_{2} s^{\prime}$ with $s v_{2} s^{\prime}>v_{2}$. In either cases, we see that $\eta\left(v_{2}, a_{2}\right) \geqslant v_{1}$.

Case 3: $a_{1}=s s^{\prime}$ and $a_{2}=s s^{\prime}$. Then either $\eta\left(v_{1}, a_{1}\right)=v_{1}$ with $v_{1}>s v_{1} s^{\prime}$ or $\eta\left(v_{1}, a_{1}\right)=s v_{1} s^{\prime}$ with $s v_{1} s^{\prime}>v_{1}$. We also have either $\eta\left(v_{2}, a_{2}\right)=v_{2}$ with $v_{2}>s v_{2} s^{\prime}$ or $\eta\left(v_{2}, a_{2}\right)=s v_{2} s^{\prime}$ with $s v_{2} s^{\prime}>v_{2}$. We break up this case into four subcases.

Subcase 3-1: $\eta\left(v_{1}, a_{1}\right)=v_{1}$ and $\eta\left(v_{2}, a_{2}\right)=v_{2}$. Then, $\eta\left(v_{1}, a_{1}\right) \leqslant \eta\left(v_{2}, a_{2}\right)$.

Subcase 3-2: $\eta\left(v_{1}, a_{1}\right)=v_{1}$ and $\eta\left(v_{2}, a_{2}\right)=s v_{2} s^{\prime}$. Then, $\eta\left(v_{1}, a_{1}\right)=v_{1} \leqslant v_{2}<s v_{2} s^{\prime}=$ $\eta\left(v_{2}, a_{2}\right)$.

Subcase 3-3: $\eta\left(v_{1}, a_{1}\right)=s v_{1} s^{\prime}$ and $\eta\left(v_{2}, a_{2}\right)=v_{2}$. Then, $\eta\left(v_{1}, a_{1}\right)=s v_{1} s^{\prime} \leqslant v_{2}=$ $\eta\left(v_{2}, a_{2}\right)$ by the lifting property.

Subcase 3-4: $\eta\left(v_{1}, a_{1}\right)=s v_{1} s^{\prime}$ and $\eta\left(v_{2}, a_{2}\right)=s v_{2} s^{\prime}$. Then, $\eta\left(v_{1}, a_{1}\right)=s v_{1} s^{\prime} \leqslant s v_{2} s^{\prime}=$ $\eta\left(v_{2}, a_{2}\right)$ by the lifting property.

For every $v \in[u, w]$ with $s v s^{\prime}<v$, notice that the image of the elements $(v, e)$, $\left(s v s^{\prime}, s s^{\prime}\right),\left(v, s s^{\prime}\right)$ under the map $\eta$ is the single element $v$. From this observation, we let $v_{1}, v_{2}, \ldots, v_{k}$ be a linear ordering of the elements of the set $Z=\left\{v: u<v<w, s v s^{\prime}<v\right\}$ such that the ranks (or lengths) of elements are weakly increasing, in other words, $\ell\left(v_{1}\right) \leqslant$ $\ell\left(v_{2}\right) \leqslant \ldots \leqslant \ell\left(v_{k}\right)$. Define posets $Q_{i}$ for $0 \leqslant i \leqslant k$ recursively as follows. Let $Q_{0}=$ $[u, w] \times\left[e, s s^{\prime}\right]$. Let $Q_{i}$ to be the poset obtained by zipping $\left\{\left(v_{i}, e\right),\left(s v_{i} s^{\prime}, s s^{\prime}\right),\left(v_{i}, s s^{\prime}\right)\right\}$ in $Q_{i-1}$. In the following proposition, we show that this is indeed a proper zipping.

Proposition 24. The triples $\left\{\left(v_{i}, e\right),\left(s v_{i} s^{\prime}, s s^{\prime}\right),\left(v_{i}, s s^{\prime}\right)\right\}$ in $Q_{i-1}$ for $1 \leqslant i \leqslant k$ are zippers.

Proof. First, we claim that $\left(v_{i}, e\right),\left(s v_{i} s^{\prime}, s s^{\prime}\right)$ and $\left(v_{i}, s s^{\prime}\right)$ are elements of $Q_{i-1}$. The element $\left(v_{i}, s s^{\prime}\right)$ has not been deleted yet, and we have not identified $\left(v_{i}, s s^{\prime}\right)$ with any element because it is at a rank higher than we have yet made identifications. The only elements ever deleted are of the form $\left(x, s s^{\prime}\right)$ where $x>s x s^{\prime}$, so $\left(v_{i}, e\right)$ and $\left(s v_{i} s^{\prime}, s s^{\prime}\right)$ have not been deleted. The only identification one could make involving $\left(v_{i}, e\right)$ and $\left(s v_{i} s, s s^{\prime}\right)$ is to identify them to each other, and that has not happened yet, and thus the claim is proved. Second, we check the conditions in the definition of a zipper. Suppose $(x, a)<$ $\left(v_{i}, e\right)$, then $x<v_{i}$ and $a=e$. Then $x<s x s^{\prime}$; otherwise the triple $\left\{\left(s x s^{\prime}, e\right),\left(x, s s^{\prime}\right),(x, e)\right\}$ is a zipper in $Q_{i-1}$ with $\ell(x)<\ell\left(v_{i}\right)$, which is impossible. Then we have $x \leqslant s v_{i} s^{\prime}$ by lifting property and thus $(x, a)<\left(s v_{i} s^{\prime}, s s^{\prime}\right)$. Now suppose $(x, a)<\left(s v_{i} s^{\prime}, s s^{\prime}\right)$; then either $a=e$ or $a=s s^{\prime}$. If $a=e$ then $(x, a)<\left(v_{i}, e\right)$ since $x \leqslant s v_{i} s^{\prime}<v_{i}$. Assume $a=s s^{\prime}$. Then $x<s v_{i} s^{\prime}$ which implies $\ell(x)<\ell\left(s v_{i} s^{\prime}\right)$ so $x$ is not $v_{r}$ for any $r \leqslant$ $i$, and then $x<s x s^{\prime}$. Then the triple $\left\{\left(x, s s^{\prime}\right),\left(s x s^{\prime}, e\right),\left(s x s^{\prime}, s s^{\prime}\right)\right\}$ is a zipper with $\ell\left(s x s^{\prime}\right)<\ell\left(v_{i}\right)$ in $Q_{i-1}$, which is impossible. Hence the triple satisfies the first condition $\left\{(x, a):(x, a)<\left(v_{i}, e\right)\right\}=\left\{(x, a):(x, a)<\left(s v_{i} s^{\prime}, s s^{\prime}\right)\right\}$. The second condition is obvious 


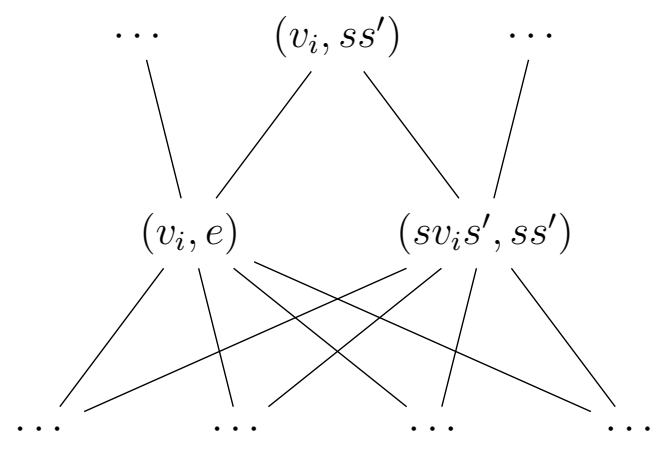

because $\left(v_{i}, e\right) \lessdot\left(v_{i}, s s^{\prime}\right)$ and $\left(s v_{i} s^{\prime}, s s^{\prime}\right) \lessdot\left(v_{i}, s s^{\prime}\right)$. Assume $(x, a)$ is covered by $\left(v_{i}, s s^{\prime}\right)$. If $a=e$ then $x=v_{i}$, so $(x, a)=\left(v_{i}, e\right)$. If $a=s s^{\prime}$ then $x \lessdot v_{i}$. Since $s v_{i} s^{\prime}<v_{i}$ and $x<s x s^{\prime}$, by the lifting property $x \leqslant s v_{i} s^{\prime}$ which implies $x=s v_{i} s^{\prime}$. Hence $\left(v_{i}, s s^{\prime}\right)$ covers no other element than $\left(v_{i}, e\right)$ and $\left(s v_{i} s^{\prime}, s s^{\prime}\right)$, and thus the third condition holds. Therefore, the triple $\left\{\left(v_{i}, e\right),\left(s v_{i} s^{\prime}, s s^{\prime}\right),\left(v_{i}, s s^{\prime}\right)\right\}$ is a zipper in $Q_{i-1}$.

In terms of zipper and operation zip, we can think of the image of $[u, w] \times\left[e, s s^{\prime}\right]$ under the map $\eta$ as a sequence of zipping operations of the triples $\left\{\left(v_{i}, e\right),\left(s v_{i} s^{\prime}, s s^{\prime}\right),\left(v_{i}, s s^{\prime}\right)\right\}$ for $v_{i} \in Z$.

Example 25. Let $u=e, w=s_{1} s_{3} s_{2} s_{6} s_{7} s_{5}$ in $\mathcal{M P}_{4}$ and let $s=s_{2}$ and $s^{\prime}=s_{6}$. Then, $u<s^{\prime} s^{\prime}, w<s w s^{\prime}$ and $u<w$. The Hasse diagram of $[u, w] \times\left[e, s s^{\prime}\right]$ is

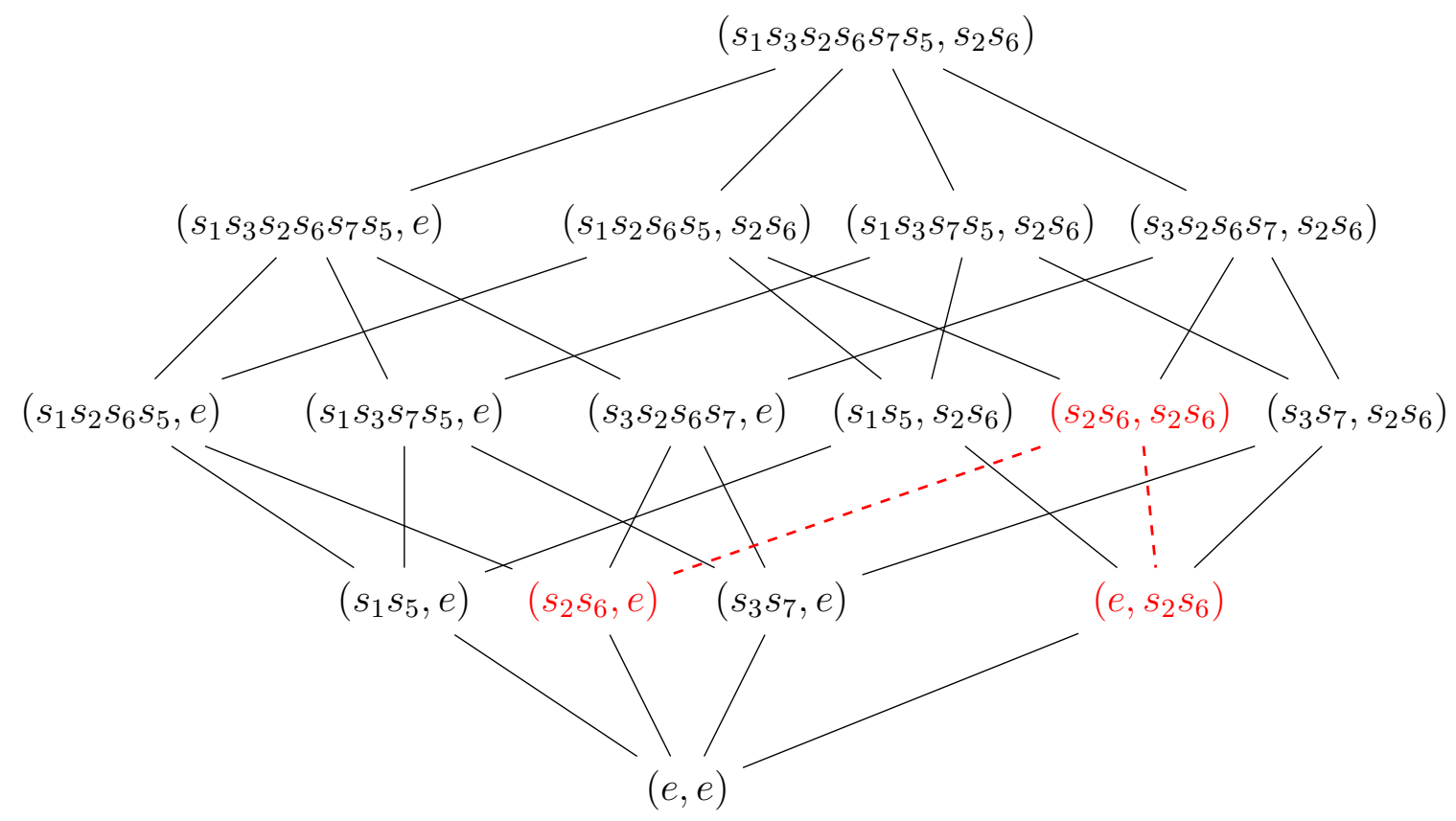

We consider the map $\eta:[u, w] \times\left[e, s s^{\prime}\right] \rightarrow\left[u, s w s^{\prime}\right]$. After zipping the zipper $\left\{\left(s_{2} s_{6}, e\right)\right.$, $\left.\left(e, s_{2} s_{6}\right),\left(s_{2} s_{6}, s_{2} s_{6}\right)\right\}$, we get 


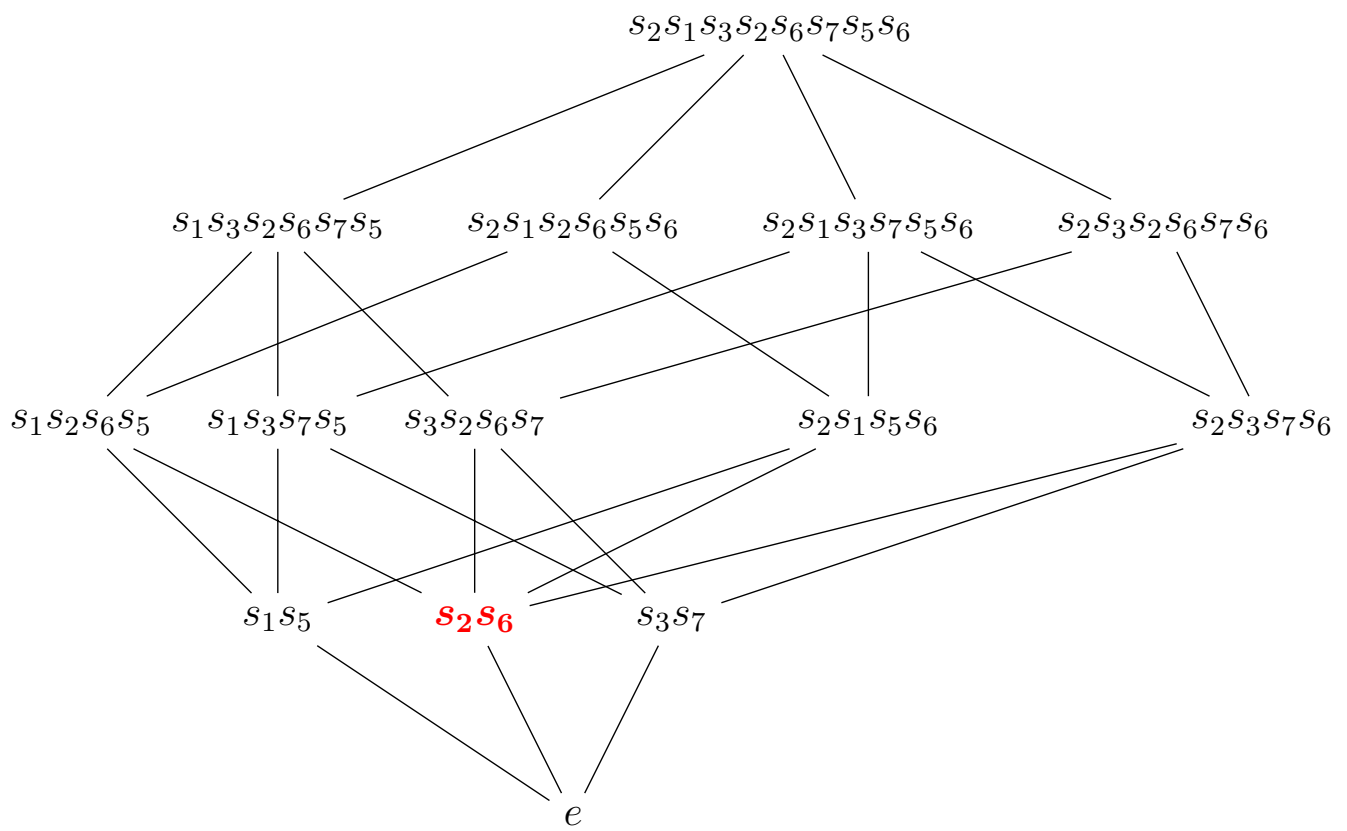

As the following proposition is an analogue of Proposition 5.2 in [13], we omit the proof.

Proposition 26. Let $u<s u s^{\prime}$ and $w<s w s^{\prime}$ and sus $\nless$ $w$. Then svs' $>v$ for all $v \in[u, w]$, and $\eta$ is an order-preserving bijection.

Proposition 26 directily implies the following corollary.

Corollary 27. Let $u<$ sus $^{\prime}$ and $w<$ sws $^{\prime}$ and sus $\nless$ s. Then the map $\theta:[u, w] \rightarrow$ $\left[\right.$ sus $^{\prime}$, sws $\left.s^{\prime}\right]$ with $\theta(v)=s v s^{\prime}$ is an order-preserving bijection.

In other words, if the condition in Corollary 27 holds, then the map $\theta$ makes a copy $\left[s u s^{\prime}, s w s^{\prime}\right]$ of the interval $[u, w]$.

We have proven the following theorem which is an analogue of Theorem 5.5 in [13].

Theorem 28. Let $w<s w s^{\prime}$ and $u<s u s^{\prime}$ and $u \leqslant w$. If sus $\notin[u, w]$ then $[u$, sws $] \cong$ $[u, w] \times\left[1, s s^{\prime}\right]$ and $\left[\right.$ sus $\left.^{\prime}, s w s^{\prime}\right] \cong[u, w]$. If sus $\in[u, w]$, then $\left[u\right.$, sws $\left.^{\prime}\right]$ can be obtained from $[u, w] \times\left[1, s s^{\prime}\right]$ by a sequence of zippings.

Now we are ready to prove Theorem 18 . This proof is essentially the same as the proof of Theorem 6.1 in [13]. Here we reproduce this proof for completeness.

Proof of Theorem 18. Part (1) follows from Proposition 26 and Corollary 27. So we focus on part (2), in the case of $s u s^{\prime} \in[u, w]$. Define the posets $Q_{i}$ as in Proposition 24. By Theorem 15,

$$
\Phi_{Q_{i-1}}-\Phi_{Q_{i}}=\Phi_{\left[(u, e),\left(v_{i}, e\right)\right]} \cdot d \cdot \Phi_{\left[\left(v_{i}, s s^{\prime}\right),\left(w, s s^{\prime}\right)\right]} \cdot
$$


where intervals in the right hand side are in $Q_{i-1}$. Since $Q_{k}=\left[u, s w s^{\prime}\right]$, sum from $i=1$ to $i=k$ to obtain

$$
\Phi_{\left[u, s w s^{\prime}\right]}=\Phi_{Q_{0}}-\sum_{i=1}^{k} \Phi_{\left[(u, e),\left(v_{i}, e\right)\right]} \cdot d \cdot \Phi_{\left[\left(v_{i}, s s^{\prime}\right),\left(w, s s^{\prime}\right)\right]} .
$$

where intervals in right hand side are in $Q_{i-1}$. Notice that the interval $\left[(u, e),\left(v_{j}, e\right)\right]$ in $Q_{j-1}$ is isomorphic to the interval $\left[(u, e),\left(v_{j}, e\right)\right]$ in $Q_{0}$ which is also isomorphic to $\left[u, v_{j}\right]$. Similarly, the interval $\left[\left(v_{j}, s s^{\prime}\right),\left(w, s s^{\prime}\right)\right]$ in $Q_{j-1}$ is isomorphic to the interval $\left[\left(v_{j}, s s^{\prime}\right),\left(w, s s^{\prime}\right)\right]$ in $Q_{0}$ which is isomorphic to $\left[v_{j}, w\right]$, and the second part of the theorem is proved.

Therefore, we have a recurrence relation for the cd-indices $\Phi(c, d)$ of intervals in $P_{n}$, or equivalently, $\mathcal{M P}_{n}$. Then we also have a recurrence relation for the ab-indices $\Psi(a, b)$ of the intervals by the relation $\Psi(a, b)=\Phi(a+b, a b+b a)$. Recall that poset $\hat{P}_{n}$ is $P_{n}$ with a unique minimum element $\hat{0}$ adjoined. We prove the following proposition which helps us compute ab-indices of intervals $[\hat{0}, \tau]$ in $\hat{P}_{n}$ recursively.

Proposition 29. Let $P$ be a graded poset of rank $n$ with a unique maximum element $\hat{1}$ and multiple minimal elements such that every interval in $P$ is Eulerian. Let $\hat{P}$ be the poset $P$ with a unique minimum element $\hat{0}$ adjoined, where $\rho(\hat{0})=-1$. If $\hat{P}$ is Eulerian, then the ab-index of $\hat{P}$ is given by

$$
\Psi_{\hat{P}}(a, b)=(a-b)^{n}+\sum_{i=0}^{n-1}(a-b)^{i} b \sum_{\rho(x)=i} \Psi_{[x, \hat{1}]}(a, b) .
$$

Proof. By the definition of ab-index and the definition of flag $f$-vector and $h$-vector,

$$
\begin{aligned}
\Psi_{\hat{P}}(a+b, b) & =\sum_{S \subseteq[n-1] \cup\{0\}} \alpha_{P}(S) u_{S} \\
& =\sum_{\hat{0}<t_{1}<\cdots<t_{k-1}<\hat{1}} a^{\rho\left(\hat{0}, t_{1}\right)-1} b a^{\rho\left(t_{1}, t_{2}\right)-1} b \ldots b a^{\rho\left(t_{k-1}, \hat{1}\right)-1},
\end{aligned}
$$

and thus

$$
\Psi_{\hat{P}}(a, b)=\sum_{\hat{0}<t_{1}<\cdots<t_{k-1}<\hat{1}}(a-b)^{\rho\left(\hat{0}, t_{1}\right)-1} b(a-b)^{\rho\left(t_{1}, t_{2}\right)-1} b \ldots b(a-b)^{\rho\left(t_{k-1}, \hat{1}\right)-1} .
$$

We rearrange this summation in terms of $t_{1}$, the lowest element in the chain except $\hat{0}$. If there is no $t_{1}$ in the chain, then the summand will be $(a-b)^{n}$. If $\rho\left(t_{1}\right)=i \in$ $\{0,1, \ldots, n-1\}$, then the summand will be $(a-b)^{i} b\left[(a-b)^{\rho\left(t_{1}, t_{2}\right)-1} b \ldots b(a-b)^{\rho\left(t_{k-1}, \hat{1}\right)-1}\right]$. By this observation, we can write (4) as

$$
\begin{aligned}
\Psi_{\hat{P}}(a, b) & =(a-b)^{n}+\sum_{i=0}^{n-1}(a-b)^{i} b \sum_{t_{1}<\cdots<t_{k-1}<\hat{1}}(a-b)^{\rho\left(t_{1}, t_{2}\right)-1} b \ldots b(a-b)^{\rho\left(t_{k-1}, \hat{1}\right)-1} \\
& =(a-b)^{n}+\sum_{i=0}^{n-1}(a-b)^{i} b \sum_{\rho\left(t_{1}\right)=i} \Psi_{\left[t_{1}, \hat{1}\right]}(a, b)
\end{aligned}
$$


as desired.

The previous proposition directly implies the following theorem. We have recursions for the ab-indices of the poset $\hat{P}_{n}$ and its intervals $[\hat{0}, \tau]$.

Theorem 30. The ab-index of $\hat{P}_{n}$ is recursively given by

$$
\Psi_{\hat{P}_{n}}(a, b)=(a-b)^{\left(\begin{array}{c}
n \\
2
\end{array}\right)}+\sum_{i=0}^{\left(\begin{array}{c}
n \\
2
\end{array}\right)-1}(a-b)^{i} b \sum_{\ell(x)=2\left(\begin{array}{c}
n \\
2
\end{array}\right)-2 i} \Psi_{[e, x]}(a, b)
$$

where $x \in \mathcal{M P}_{n}$. Let $\tau \in P_{n}$ such that $c(\tau)=k \leqslant\left(\begin{array}{l}n \\ 2\end{array}\right)$ and $\phi(\tau)=w \in \mathcal{M P}_{n}$. The ab-index of the interval $[\hat{0}, \tau] \subset \hat{P}_{n}$ is recursively given by

$$
\Psi_{[\hat{0}, \tau]}(a, b)=(a-b)^{k}+\sum_{i=0}^{k-1}(a-b)^{i} b \sum_{\substack{x: x>w \\
\ell(x)=2\left(\begin{array}{c}
n \\
2
\end{array}\right)-2 i}} \Psi_{[w, x]}(a, b) .
$$

\section{Acknowledgements}

The author wishes to thank his advisor, Susanna Fishel, for her many helpful comments. This paper could not be finished without her guidance. The author would also like to thank Thomas Lam for introduction to the uncrossing posets and the problems presented in this paper. The author is also grateful to the Simons Foundation for travel support (Grant 359602, PI S.Fishel) for participating in FPSAC 2016.

\section{References}

[1] Joshua Alman, Carl Lian, and Brandon Tran. Circular planar electrical networks: Posets and positivity. Journal of Combinatorial Theory, Series A, 132:58 - 101, 2015.

[2] Margaret M. Bayer and Louis J. Billera. Counting faces and chains in polytopes and posets. In Combinatorics and algebra (Boulder, Colo., 1983), volume 34 of Contemporary Mathematics, pages 207-252. Amer. Math. Soc., Providence, RI, 1984.

[3] Margaret M. Bayer and Andrew Klapper. A new index for polytopes. Discrete 6 Computational Geometry, 6(1):33-47, 1991.

[4] Anders Björner and Francesco Brenti. Combinatorics of Coxeter groups, volume 231 of Graduate Texts in Mathematics. Springer, New York, 2005.

[5] Yves Colin de Verdière, Isidoro Gitler, and Dirk Vertigan. Réseaux électriques planaires. II. Commentarii Mathematici Helvetici, 71(1):144-167, 1996.

[6] E.B. Curtis, D. Ingerman, and J.A. Morrow. Circular planar graphs and resistor networks. Linear Algebra and its Applications, 283(1):115-150, 1998.

[7] Richard Ehrenborg and Margaret Readdy. Coproducts and the cd-index. Journal of Algebraic Combininatorics, 8(3):273-299, 1998. 
[8] Yu-Tin Huang, Congkao Wen, and Dan Xie. The positive orthogonal Grassmannian and loop amplitudes of ABJM. Journal of Physics A: Mathematical and Theoretical, 47(47):474008, 2014.

[9] R. Kenyon. The Laplacian on planar graphs and graphs on surfaces. In Current developments in mathematics, 2011, pages 1-55. Int. Press, Somerville, MA, 2012.

[10] Joonho Kim and Sangmin Lee. Positroid stratification of orthogonal Grassmannian and ABJM amplitudes. Journal of High Energy Physics, (9):085, front matter+30, 2014.

[11] Thomas Lam. The uncrossing partial order on matchings is Eulerian. Journal of Combinatorial Theory, Series A, 135:105 - 111, 2015.

[12] Thomas Lam. Electroid varieties and a compactification of the space of electrical networks. Advances in Mathematics, 338:549-600, 2018.

[13] Nathan Reading. The cd-index of Bruhat intervals. Electronic Journal of Combinatorics, 11(1):\#R74, 25, 2004.

[14] Richard P. Stanley. Balanced Cohen-Macaulay complexes. Transactions of the American Mathematical Society, 249(1):139-157, 1979.

[15] Richard P. Stanley. Flag $f$-vectors and the cd-index. Mathematische Zeitschrift, 216(3):483-499, 1994.

[16] Richard P. Stanley. Enumerative combinatorics. Volume 1, volume 49 of Cambridge Studies in Advanced Mathematics. Cambridge University Press, Cambridge, second edition, 2012. 